\title{
PROCEDIMENTO DE DISSOCIAÇÃO DE PROCESSOS
}

\author{
Mário B. Ferreira ${ }^{12}$ \\ Joana Reis ${ }^{1}$ \\ Diana Orghian ${ }^{1}$ \\ Jerônimo Sôro ${ }^{1}$
}

[Enviado a 06-01-2013. Aceite a 12-06-2013]

Resumo: O procedimento de dissociação de processos (PDP) é um paradigma metodológico que tem vindo a ser largamente usado em várias áreas da investigação em psicologia. A principal inovação deste procedimento resulta da possibilidade que fornece ao investigador de dissociar e quantificar o grau de automaticidade e de controlo subjacentes ao comportamento humano no desempenho de diversas tarefas. Este procedimento, que incorpora em si mesmo uma concepção própria de automatismo e controlo, é um contributo importante para a resolução de problemas metodológicos associados aos testes directos e indirectos e às dissociações de processos baseadas em tarefas. Na presente exposição faz-se uma descrição pormenorizada do paradigma em causa recorrendo a exemplos específicos de vários domínios da Psicologia (e.g., memória, estereótipos, julgamento e decisão). São também mencionados os pressupostos básicos de aplicação do PDP e por fim referem-se as limitações do paradigma, cuidados a ter na sua aplicação assim como alguns procedimentos alternativos.

Palavras-chave: Procedimento de dissociação de processos (PDP), automaticidade, controlo.

Process-dissociation procedure (Abstract): The process-dissociation procedure (PDP) is a methodological paradigm widely used in different domains of psychological research. The main innovation of this procedure stems from the possibility it offers to dissociate and to quantify the degree of automaticity and control underlying human performance in different tasks. The PDP embodies a particular definition of automaticity and control, which is an important contribution to solve

\footnotetext{
${ }^{1}$ Faculdade de Psicologia, Universidade de Lisboa, Portugal

2 A correspondência relativa a este artigo deverá ser enviada para Mário Boto Ferreira, Faculdade de Psicologia, Universidade de Lisboa, Alameda da Universidade 1649-013; E-mail: mferreira@fp.ul.pt.
} 
methodological problems related with direct and indirect tests and with dissociation of processes based on tasks. In the present paper the paradigm will be described using several examples from different areas of psychological research (e.g., memory, stereotypes, judgment and decision making). The basic assumptions underlying the PDP, guidelines to its application, the PDP main limitations and some alternative procedures will also be described.

Keywords: Process-dissociation procedure (PDP), automaticity, control.

\section{Introdução}

Até que ponto podemos controlar a forma como processamos informação? Até que ponto este processamento de informação decorre de estratégias intencionais e conscientes ou, pelo contrário, ocorre de forma largamente inconsciente?

Responder a estas questões é simultaneamente crucial para a investigação em psicologia e extremamente difícil de um ponto de vista metodológico.

Para enfrentar estas dificuldades, Jacoby (1991, 1998; Jacoby, Toth, \& Yonelinas, 1993) desenvolveu um paradigma metodológico inovador conhecido por procedimento de dissociação de processos (PDP) que permitiu não só dissociar mas também estimar matematicamente os contributos de processos controlados e de processos automáticos no desempenho de diferentes tarefas.

Inicialmente proposto no âmbito de estudos de memória, a aplicação do PDP foi mais tarde generalizada (com pequenas modificações) a outros domínios da cognição humana como atitudes e estereótipos (e.g., Payne, 2001; 2005), percepção social e inferências de traços de personalidade (Ferreira et al., 2012), julgamento e decisão em condições de incerteza (Ferreira, Garcia-Marques, Sherman \& Sherman, 2006), julgamentos morais (Conway \& Garownski, 2013), entre outros. Hoje em dia, mais do que um procedimento especificamente desenhado para dissociar memória explícita de memória implícita, o PDP é um paradigma metodológico geral que incorpora uma teoria de automatismo e controlo própria e que pode ser usado para distinguir o contributo relativo de processos controlados e automáticos nos mais diversos domínios da investigação experimental em psicologia. É justamente nesta dimensão de paradigma metodológico geral, que o PDP ganha toda a sua real importância para a investigação em psicologia.

Neste artigo, começamos por identificar e discutir brevemente o principal problema metodológico ao qual Jacoby (1991) pretendeu responder com a introdução do PDP. Seguidamente são descritas sucintamente as pri- 
meiras aplicações na investigação em memória. Depois de revermos as origens do PDP, focar-nos-emos nas suas características metodológicas gerais, pressupostos teóricos e limitações, uma vez que pensamos serem estes os aspectos chave que um cientista que considere recorrer ao PDP deve conhecer, independentemente da sua área específica de investigação.

\section{As origens do PDP: o pressuposto da tarefa "pura"}

Embora a literatura científica em psicologia experimental seja pródiga em exemplos onde estratégias ou processos intencionais são usados para explicar os resultados, há muito que também sabemos que a memória humana tem uma natureza associativa cujo funcionamento ocorre largamente fora do controlo estratégico do indivíduo (Anderson \& Bower, 1973).

Curiosamente, só a partir de meados dos anos 70 é que a investigação e teorização em psicologia cognitiva começaram a lidar directamente com a diferença entre processamento de informação automático (largamente involuntário) e controlado (maioritariamente voluntário) (e.g., Posner \& Snyder, 1975; Schneider \& Shiffrin, 1977; Shiffrin \& Schneider, 1977).

De acordo com a visão clássica de automaticidade, os processos cognitivos automáticos são caracterizados pela activação espontânea de associações pré-existentes ou estruturas de conhecimento desenvolvidas ao longo do tempo por activação repetida em memória. A activação destas estruturas acontece sem qualquer consciência por parte da pessoa, sendo muitas vezes também inconsciente a sua influência nos comportamentos, ideias, julgamentos e atitudes da mesma. Uma outra característica dos processos automáticos está relacionada com a sua falta de intencionalidade, uma vez que são desencadeados por pistas externas ou internas ao indivíduo mas tipicamente independentes da sua vontade (Neely, 1977; Shiffrin \& Dumais, 1981). Trata-se de processos altamente eficientes que uma vez desencadeados tendem a decorrer de forma obrigatória, não fazendo uso (ou fazendo um uso muito modesto) de recursos cognitivos gerais disponíveis. Por último e em consonância com o que foi dito anteriormente estes processos ocorrem fora do controlo do sujeito e são dificilmente controláveis por este (Bargh, 1994).

Em contraste, os processos cognitivos controlados são tipicamente descritos como sendo intencionais e requerendo a atenção focalizada do indivíduo. Há habitualmente um acesso introspectivo consciente por parte da pessoa que acompanha o desencadeamento e evolução destes processos. Como o nome indica são processos que se encontram sob o controlo da pessoa, desde que esta disponha dos recursos cognitivos necessários para exercer tal controlo. Assim, embora limitados pela capacidade da memória de 
trabalho, são flexíveis podendo por isso convergir com os processos automáticos, ou pelo contrário, opor-se a estes.

Note-se contudo que uma caracterização dicotómica entre processos automáticos e controlados com base nas características acima apresentadas é provavelmente forçada. Uma perspectiva mais em linha com a investigação acumulada sugere um contínuo entre controlo e automaticidade onde o processamento de informação, em cada circunstância, pode possuir mais ou menos características de automaticidade (ver Wegner \& Bargh, 1998).

A investigação experimental decorrente da abordagem dualista acima referida tem vindo a sugerir que processos cognitivos automáticos e controlados podem operar independentemente um do outro (Logan, 1980; Logan \& Cowan, 1984; Neely, 1977; Posner \& Snyder, 1975). Tal conclusão decorre da obtenção de dissociações entre o desempenho das pessoas em tarefas que medem respostas decorrentes do processamento automático da informação e o seu desempenho em tarefas que medem respostas controladas.

Um exemplo deste tipo de dissociações são os resultados obtidos em testes directos e indirectos de memória (para uma revisão ver Hintzman, 1990). Num teste directo (ou explícito) de memória (e.g., teste de recordação livre ou de reconhecimento) é pedido aos participantes que recordem informação passada. Pelo contrário, num teste indirecto (ou implícito) de memória é pedido aos participantes que realizem uma tarefa capaz de reflectir indirectamente a memória da informação passada. Um exemplo deste tipo de tarefa é pedir aos participantes que leiam uma lista de palavras durante a fase de estudo (e.g., "casa") e mais tarde, na fase de teste, pedir-lhes que completem fragmentos dessas palavras (e.g., "c_s_") com a primeira palavra que lhes ocorrer (e.g., Graf \& Mandler, 1984; Tulving, Schacter, \& Stark, 1982). A evidência a favor da existência de uma memória implícita das palavras estudadas, dissociada da memória explícita, resulta justamente da obtenção de resultados que mostram que, nos testes indirectos, a apresentação de palavras como "casa" na fase de estudo aumenta a probabilidade destas palavras serem usadas para completar os fragmentos (e.g., "c_s_") na fase de teste, mesmo quando em testes directos não há sinal de recordação explícita dessas, ou seja, os participantes não se recordam destas palavras como tendo sido estudadas anteriormente. Vários são os estudos que nos mostram dissociações entre estes dois tipos de memória, incluindo dados de pacientes neuropsicológicos. Sabemos por exemplo que pacientes que sofrem de amnésia (para uma revisão ver Schacter, Chiu, \& Ochsner, 1993) apresentam um desempenho pior que o dos participantes da condição controlo (i.e., não amnésicos) em tarefas que requerem o uso de estratégias deliberadas de recuperação de informação / memória explícita (e.g., recordar se uma palavra foi ou não anteriormente apresentada). No entanto em tarefas que dependem apenas de memória implícita (e.g., efeitos de primação na tarefa de 
completamento de palavras), amnésicos e participantes da condição controlo apresentam resultados semelhantes.

Tais procedimentos com base na dissociação de tarefas são também comummente usados noutras áreas da investigação em psicologia. É o caso da investigação em estereótipos e preconceito, que usa um número crescente de medidas implícitas como o teste de primação afectiva (Fazio, Jackson, Dunton, \& Williams, 1995) ou o famoso teste de associação implícita (Greenwald, McGhee, \& Schwartz, 1998). Estas medidas são muitas vezes utilizadas para substituir ou complementar medidas de preconceito explícitas, como a escala moderna de racismo (McConahay, 1986) ou o termómetro afectivo (Haddock, Zanna, \& Esses, 1993).

Contudo, de um ponto de vista metodológico, toda a evidência experimental assim obtida lida com uma limitação de fundo dificilmente ultrapassável por este tipo de procedimento: o pressuposto da tarefa "pura". Trata-se da suposição de que uma dada tarefa experimental pode ser usada para medir, de forma pura, apenas processos cognitivos automáticos ou apenas processos controlados.

A crítica da identificação de processos automáticos e controlados com diferentes tarefas foi inicialmente feita por diversos autores da investigação em memória (e.g., Jacoby, 1991; Reingold \& Merikle, 1990), que notaram que o desempenho nos testes indirectos de memória é frequentemente contaminado pelo uso intencional da memória. Ou seja, nada impede os participantes de usarem a sua memória explícita das palavras estudadas na fase de estudo quando lhes é pedido para completar os fragmentos de palavras com as primeiras palavras que lhes ocorrerem. Por outro lado, é virtualmente impossível garantir que num teste directo de memória não haja contaminação proveniente de memória implícita. Num teste de reconhecimento por exemplo, os participantes podem efectivamente reconhecer uma palavra como tendo sido anteriormente apresentada ou tentar apenas adivinhar as palavras que foram ou não apresentadas. Neste último caso, a memória implícita decorrente da fase de estudo leva a que estes "adivinhem" com maior frequência palavras que foram efectivamente estudadas (devido à sua familiaridade), inflacionando assim a medida de memória explícita.

Embora tenha sido aqui ilustrado com o caso dos testes directos e indirectos de memória, o problema decorrente do pressuposto da tarefa pura tem uma natureza geral e tem sido recorrentemente revisitado e discutido em diversos domínios de investigação como em estereótipos e preconceitos (e.g., Payne, 2001, 2008) e julgamento e decisão (e.g., Bishara, 2005; Ferreira et al., 2006).

Em suma, nenhuma tarefa é uma medida "pura" de um processo cognitivo. É tecnicamente impossível garantir que qualquer resposta observável dependa inteira e somente de processamento automático ou controlado. É, 
aliás, altamente provável que a maioria do comportamento que a psicologia procura compreender decorra simultaneamente de processos automáticos e controlados que co-ocorrem e que se influenciam mutuamente (Wegner \& Bargh, 1998).

Mas então como aceder e estudar o contributo de ambos os processos controlados e automáticos?

Em 1991, Larry Jacoby propôs um procedimento de dissociação de processos (PDP) cujo objectivo é justamente a obtenção de estimativas do contributo dos processamentos controlado e automático evitando os problemas metodológicos da dissociação de tarefas acima referidos.

\section{PDP enquanto procedimento de dissociação de processos em estudos de memória}

O PDP tem por base três ideias originais que Larry Jacoby sintetizou no seu artigo seminal de 1991. A primeira ideia inovadora de Jacoby foi desenvolver tarefas onde memória implícita e explícita funcionassem em oposição (Jacoby, Kelley, Brown, \& Jasechko, 1989). Na prática, tais tarefas são equivalentes a testes indirectos de memória com instruções para não usar nenhuma das palavras de que se consiga efectivamente recordar. Por exemplo, num teste indirecto de memória, após apresentação de uma lista de palavras, pode ser pedido aos participantes para, na fase de teste, completarem fragmentos de palavras apresentadas uma a uma (algumas das quais previamente estudadas) com a primeira palavra que lhes ocorrer, sem fazer qualquer referência à lista inicialmente estudada. Jacoby e colaboradores (1993) usaram instruções semelhantes mas com uma mudança: disseram aos participantes para não completarem os fragmentos com palavras que tivessem sido previamente apresentadas. Com estas instruções a memória explícita da lista apresentada opõe-se à memória implícita. Com efeito, as vezes em que os participantes completaram os fragmentos com palavras previamente apresentadas são reveladoras de memória implícita na ausência de memória explícita (caso contrário, as palavras não seriam usadas).

Contudo, procedimentos que se baseiem simplesmente na lógica da oposição, embora permitam separar claramente memória implícita de memória explícita, funcionam como testes conservadores de memória implícita. Isto porque o desempenho dos participantes apenas revela os efeitos das influências implícitas na memória na ausência de memória explícita. Ora o facto de termos memória explícita para uma palavra não quer necessariamente dizer que não tenhamos também memória implícita. Por isto, um procedimento de oposição de processos subestima sistematicamente as influências automáticas da memória. 
A segunda ideia inovadora de Jacoby (1991) foi corrigir para esta subestimação comparando o desempenho mnésico em condições em que memória implícita e explícita se opõem e em condições em que as duas actuam de forma concertada. Na prática, testes em que memória implícita e explícita funcionam de maneira concertada são equivalentes a testes directos de memória com instruções para adivinhar quando o participante não consegue recordar explicitamente uma palavra das estudadas para completar o fragmento. Dado que este "adivinhar" é "informado" pela memória implícita que o participante tem da lista de estudo, o desempenho neste teste é o resultado combinado de memória explícita e implícita. Ou seja, o completamento dos fragmentos com palavras previamente apresentadas pode ser o resultado de memória explícita, de influências implícitas da memória ou de ambas.

Jacoby (1991) usou os termos Recolecção (R) e Familiaridade (F) para se referir respectivamente às influências explícitas e implícitas da memória. Ao procedimento com base na lógica da oposição chamou teste de exclusão e ao procedimento em que os dois processos agem concertadamente chamou teste de inclusão. A proporção de itens estudados que são usados para completar fragmentos no teste de inclusão é o resultado de $\mathrm{R}+\mathrm{F}-(\mathrm{R} \cap \mathrm{F})$. $\mathrm{O}$ termo $(\mathrm{R} \cap \mathrm{F})$, ou seja, a intersecção entre Recolecção e Familiaridade, corresponde a situações em que os participantes responderam ao problema recorrendo simultaneamente a estratégias de Recolecção e de Familiaridade (e.g., completar um fragmento com a primeira palavra que lhe ocorre e verificar que esta palavra faz parte da lista previamente estudada). Na medida em que $\mathrm{R} \cap \mathrm{F}$ é um subconjunto de $\mathrm{R}$ e também de $\mathrm{F}$, para evitar que este seja contabilizado duas vezes (uma vez em $\mathrm{R}$ e outra vez em F) torna-se necessário subtrair $\mathrm{R} \cap \mathrm{F}$ à soma $\mathrm{R}+\mathrm{F}$. A proporção de itens estudados que são usados para completar fragmentos no teste de exclusão é o resultado de $\mathrm{F}-(\mathrm{R} \cap \mathrm{F})$. $\mathrm{Ou}$ seja, corresponde ao completamento de fragmentos com base apenas na familiaridade $(F)$. Completamentos que envolvam simultaneamente $R$ e $F$ (i.e., $\mathrm{R} \cap \mathrm{F}$ ) têm que ser subtraídos uma vez que quando há recolecção da palavra estudada os participantes não vão usá-la para completar os fragmentos mesmo que a palavra seja muito familiar $(\mathrm{F})$.

Finalmente, a terceira inovação de Jacoby (1991) foi assumir (com base em resultados anteriores, ver por exemplo Mandler, 1980) uma concepção teórica em que $\mathrm{F}$ e $\mathrm{R}$ são processos de memória independentes. Isto quer dizer que a intersecção de $\mathrm{R}$ e $\mathrm{F}$ é igual ao produto de $\mathrm{R}$ por $\mathrm{F}(\mathrm{R} \cap \mathrm{F}=\mathrm{RF})$, tornando-se assim possível formalizar matematicamente a relação entre $\mathrm{F}$ e $\mathrm{R}$ (onde "P" é a probabilidade de usar itens estudados dada pela proporção de itens estudados usados na fase de teste):

$$
\begin{aligned}
& \mathrm{P}(\text { usar palavra estudada })_{\text {inclusão }}=\mathrm{R}+\mathrm{F}-\mathrm{RF}=\mathrm{R}+\mathrm{F}(1-\mathrm{R}) \\
& \mathrm{P}(\text { usar palavra estudada })_{\text {exclusão }}=\mathrm{F}-\mathrm{RF}=\mathrm{F}(1-\mathrm{R})
\end{aligned}
$$


E quantificar estas duas componentes resolvendo as equações acima:

$\mathrm{R}=\mathrm{P}(\text { usar palavra estudada })_{\text {inclusão }}-(\text { usar palavra estudada })_{\text {exclusão }}$

$\mathrm{F}=\mathrm{P}(\text { usar palavra estudada })_{\text {exclusão }} /(1-\mathrm{R})$

A primeira aplicação do PDP (Jacoby,1991; estudo 3) foi feita num estudo de memória que envolvia um teste de reconhecimento. Os participantes foram expostos numa primeira fase a palavras e anagramas apresentados sequencialmente num ecrã de computador, com instruções para ler as palavras e resolver os anagramas. Numa segunda fase era apresentada oralmente uma outra lista de palavras com instruções para repetir cada palavra em voz alta e memorizar a palavra para um teste de memória posterior. Seguia-se um teste de reconhecimento que podia ter instruções de exclusão, isto é, uma palavra deveria ser reconhecida como tendo sido previamente apresentada apenas se tivesse sido apresentada oralmente na segunda fase de estudo, mas não na primeira; ou instruções de inclusão, isto é, uma palavra deveria ser identificada como previamente apresentada quando tinha sido apresentada na primeira ou na segunda fase de estudo. $\mathrm{Na}$ medida em que apenas uma das condições (exclusão) implica o recurso a processos controlados de recolecção para distinguir entre as palavras apresentadas na primeira fase e as apresentadas na segunda fase de estudo, a diferença de desempenho entre as condições de inclusão e exclusão fornece, segundo Jacoby, uma medida de controlo consciente.

Nesta primeira aplicação do PDP as instruções de inclusão e exclusão foram manipuladas inter-participantes pelo que só foi possível estimar $\mathrm{R}$ e $\mathrm{F}$ de forma global (e não por participante) o que obviamente limita o tipo de análise estatística possível de realizar (ver Garcia-Marques \& Ferreira, no prelo). Assim, Jacoby (1991) limitou-se a comparar as estimativas obtidas neste terceiro estudo com o desempenho de outros participantes num estudo semelhante ao acima descrito, onde incluíra uma tarefa de divisão da atenção durante a codificação de palavras na fase de estudo e instruções de exclusão na fase de teste (Jacoby, 1991, estudo 2). Na medida em que esta divisão da atenção impediu a Recolecção (processo controlado) mas não afectou a Familiaridade (processo automático), o desempenho dos participantes neste estudo deveria depender sobretudo da Familiaridade. Com efeito, as estimativas de Familiaridade (calculadas no estudo 3) foram praticamente iguais ao desempenho dos participantes em condições de atenção dividida no estudo 2 (.59 e .54 para anagramas; e .42 e .43 para palavras, ver Jacoby, 1991, pp. 528-530). Este resultado que foi interpretado como uma primeira validação do PDP.

Posteriormente, Jacoby, Toth e Yonelinas (1993) realizaram novos estudos de memória utilizando o PDP mas desta vez a medida dependente usada foi a proporção de fragmentos de palavras que, na fase de teste, foram 
preenchidos com palavras previamente apresentadas na lista de estudo com instruções de inclusão e exclusão manipuladas intra-participantes. Os participantes eram instruídos para tentar recordar as palavras previamente estudadas tendo como pista fragmentos dessas palavras. Sempre que o fragmento aparecesse a verde deveriam usá-lo para recordar uma palavra estudada ou, se não se recordassem de nenhuma palavra, deviam completá-lo com a primeira palavra que lhes ocorresse (inclusão); sempre que o fragmento aparecesse a vermelho deveriam completá-lo com uma palavra que não tivesse sido previamente estudada (exclusão) ${ }^{3}$. Os participantes estudavam as listas de palavras em condições de atenção dividida ou sem atenção dividida (e.g., Jacoby et al., 1993; estudo 1b). Na condição de atenção dividida os participantes tiveram, como esperado, um pior desempenho mnésico do que os participantes sem atenção dividida. O uso do PDP permitiu verificar que tal queda do desempenho se deveu exclusivamente a uma diminuição de $\mathrm{R}$ (de .25 para .00) que virtualmente desapareceu enquanto $\mathrm{F}$ se manteve largamente invariante (.47 e .46, respectivamente). Estes estudos não só confirmaram a interpretação dos resultados de Jacoby (1991) como estabeleceram o PDP como uma forma de dissociação dos processos explícitos e implícitos (Recolecção e Familiaridade) em memória.

$\mathrm{O}$ uso do PDP nos estudos de memória não ficou por aqui tendo-se acumulado ao longo das duas últimas décadas uma quantidade impressionante de investigações com e sobre o PDP que têm permitido a sua validação convergente assim como a identificação das condições adequadas de aplicação (para uma revisão ver Yonelinas \& Jacoby, 2012).

\section{O PDP enquanto paradigma metodológico geral para dissociação de contribuições de processos controlados e automáticos}

Originalmente desenvolvido para separar as contribuições automática (Familiaridade) e controlada (Recolecção) do desempenho em tarefas de memória (Jacoby, 1991, Jacoby, Toth \& Yonelinas, 1993), o PDP é, hoje em dia, um instrumento metodológico geral capaz de separar e dissociar as con-

\footnotetext{
${ }^{3}$ A correcta compreensão e respeito por estas instruções por parte dos participantes é essencial para garantir que $\mathrm{R}$ e $\mathrm{F}$ funcionam como fontes independentes de resposta tal como preconiza o PDP. Ou seja, em cada ensaio os participantes devem sempre começar por tentar recordar uma palavra (que pode servir para completar ou não o fragmento) e só depois eventualmente responder com a primeira palavra que lhes ocorrer. O recurso a outras estratégias de resposta como por exemplo gerar uma palavra com base no fragmento e só depois tentar recordar se a palavra gerada foi previamente estudada invalida o uso do PDP porque R torna-se dependente da fase de geração que é influenciada por F (a este respeito ver Jacoby, 1998).
} 
tribuições dos processos controlados e automáticos nos mais diversos domínios.

O PDP faz uso, numa mesma tarefa, de uma condição de facilitação ou de inclusão na qual processos automáticos e controlados actuam de forma concertada convergindo numa mesma resposta; e de uma condição de interferência ou exclusão na qual os dois processos actuam em oposição, divergindo para respostas diferentes. Assumindo que ambos os processos contribuem para a resposta observada e que operam de forma independente, torna-se possível, recorrendo a álgebra simples, obter estimativas quantitativas de automatismo (A) e controlo (C) comparando o desempenho dos participantes nas duas condições de inclusão e exclusão.

A título de ilustração, imagine uma pessoa que tem uma forte tentação por doces e que resolve durante a semana fazer dieta e não comer doces nenhuns, enquanto durante o fim-de-semana come todos os doces que lhe apetecer. Digamos ainda que durante os fins-de-semana, e após observação do seu comportamento, concluímos que a probabilidade desta pessoa comer um doce é de 0.9. Esta probabilidade tanto pode resultar da tentação dificilmente controlável e largamente automática (A) de comer doces como da intenção explícita e controlada de comer doces (C). Durante o resto da semana a mesma pessoa tem uma probabilidade de .2 de comer doces. $\mathrm{Ou}$ seja, ela claramente controla a sua tentação por doces ainda que este controlo não seja perfeito. Esta probabilidade de 0.2 resulta assim da sua tentação por doces (A) quando o controlo falha $(1-\mathrm{C})$. Ora, se subtrairmos 0.2 a 0.9 obteremos uma boa estimativa da sua capacidade de se controlar: 0.7. Assumindo que ela só come doces durante a semana quando o seu controlo falha ${ }^{4}$, isto quer dizer que a probabilidade de comer doces durante a semana (0.2) é igual à tentação automática na ausência de controlo, ou seja: $\mathrm{A}(1-\mathrm{C})$. É agora fácil chegar a uma estimativa de $\mathrm{A}$, pois se $\mathrm{A}(1-\mathrm{C})=0.2$ então $\mathrm{A}=0.2 /(1-$ C). Como já sabemos que $\mathrm{C}=0.7$, então $\mathrm{A}=0.2 /(1-0.7) \Leftrightarrow \mathrm{A}=0.67$. $\mathrm{Ou}$ seja, trata-se de uma tentação relativamente forte que só não se traduz mais vezes em comer doces durante a semana devido à inibição intencional que a pessoa do nosso exemplo exerce sobre esta tendência comportamental automática (para ilustrações semelhantes ver Payne \& Bishara, 2009; Shiv \& Fedorikhin, 1999).

Assim temos as seguintes equações para o mesmo comportamento (e.g., comer doces) em condições de inclusão (e.g., comer doces ao fim de semana) e em condições de exclusão (e.g., comer doces durante a semana):

\footnotetext{
${ }^{4}$ É possível assumir outro tipo de relação entre A e C. Designadamente, assumir que C só ocorre na ausência de A. Contudo o seu uso na investigação com modelos multinomiais semelhantes ao PDP tem sido relativamente raro e tem estado confinado a domínios muito específicos (a este respeito ver Payne \& Bishara, 2009).
} 


$$
\begin{aligned}
& \text { Comportamento }_{\text {Inclusão }}=\mathrm{C}+\mathrm{A}(1-\mathrm{C}) \\
& \text { Comportamento }_{\text {Exclusão }}=\mathrm{A}(1-\mathrm{C})
\end{aligned}
$$

Imaginemos agora que distraímos a atenção da pessoa acima referida. É natural que ela coma mais doces quando está distraída...mas porquê? Porque reduzimos a sua capacidade de exercer controlo $(\mathrm{C})$ ? Ou porque aumentamos a sua tentação automática (A) por doces (o que poderia acontecer se o que tivéssemos usado para a distrair fossem justamente...doces)?

Só um procedimento como o PDP nos permite examinar experimentalmente questões como estas. Ou seja, verificar qual o contributo específico dos processos controlados e automáticos que estão subjacentes ao comportamento de comer doces em função de diversas manipulações.

$\mathrm{O}$ uso das equações acima implica, no entanto, criar condições experimentais onde se define operacionalmente Controlo ou processamento controlado como levando ao output considerado subjectivamente certo ou intencionalmente desejado (e.g., não comer doces durante a semana). Decorre desta definição de Controlo a própria definição operacional de automaticidade ou processamento automático: o que leva ao output oposto ao da resposta intencional quando o Controlo falha (e.g., comer doces durante a semana). Importa contudo salientar que estas definições devem ser enquadradas pela própria lógica que subjaz ao PDP, não reflectindo nenhuma posição de princípio que implique que as respostas decorrentes de processamento automático sejam inerentemente piores do que as respostas controladas.

Em suma, através da obtenção de estimativas de A e C numa mesma tarefa, o PDP permite o teste empírico de dissociações de processos que decorrem teoricamente das características conceptuais de ambos os processos.

\section{Pressupostos do PDP}

Como qualquer tarefa ou procedimento experimental, o PDP assenta em pressupostos que, se violados, põem em causa a interpretação das estimativas de A e C. O principal pressuposto é o de independência entre os processos controlados e automáticos. As duas expressões algébricas apresentadas acima, que equacionam o desempenho nas condições de inclusão e exclusão em termos dos dois processos, expressam matematicamente esta independência. Neste sentido, violações de independência invalidam o PDP. Para além disto o PDP assume ainda que A e C são iguais nas condições de inclusão e de exclusão. Ou seja, a preponderância dos dois modos de processamento não muda em função de se tratar de um ensaio de inclusão (onde ambos os processos contribuem para uma mesma resposta) ou de exclusão 
(onde o processo controlado passa a opor-se ao automático). Como o PDP envolve apenas duas medidas empíricas (desempenho nas condições de inclusão e exclusão) para estimar dois parâmetros: $\mathrm{A}$ e $\mathrm{C}$, sempre que $\mathrm{A}_{\text {inclusão }}$ $\neq \mathrm{A}_{\text {exclusão }}$ e/ou $\mathrm{C}_{\text {inclusão }} \neq \mathrm{C}_{\text {exclusão }}$ torna-se matematicamente inviável obter as estimativas de A e C, invalidando, nestes casos, o uso do PDP ${ }^{5}$. A título ilustrativo, voltemos ao exemplo dos doces, se a tentação automática para comer doces durante o fim-de-semana (condição de inclusão) for menor do que tentação automática de comer doces durante a semana (condição de exclusão) então isto quer dizer que a componente automática A que se assume ser a mesma nas respectivas equações de inclusão e exclusão (ver fórmulas acima) é afinal diferente. Passa a haver uma componente automática para a inclusão, A1, que é menor do que a componente automática para a condição de exclusão, A2. Em circunstâncias como esta passaríamos a ter três incógnitas: A1, A2, e C para calcular a partir de duas equações de $1^{\circ}$ grau, o que é impossível. O mesmo se aplica quando a componente $\mathrm{C}$ é diferente nas condições de inclusão e exclusão.

Pela mesma razão (i.e., apenas duas medidas empíricas para estimar dois parâmetros) o PDP não possui graus de liberdade extra, o que limita a realização de testes directos dos pressupostos, i.e., análises de fit das estimativas dos parâmetros possíveis de realizar com modelos polinomais mais complexos (mas ver a este respeito Jacoby, 1998; Yonelinas \& Jacoby, 2012)6. A garantia de que os pressupostos básicos do PDP não estão a ser violados pode, contudo, ser obtida experimentalmente mostrando-se que certas manipulações, histórica e teoricamente, identificadas com um dos processos afecta selectivamente esse processo deixando o outro invariante. Por exemplo, uma das marcas características dos processos automáticos é a sua elevada eficiência, ou seja, não usam recursos cognitivos gerais, enquanto os processos controlados dependem da disponibilidade de recursos cognitivos. Neste sentido, previu-se que manipulações de sobrecarga cognitiva que comparassem o desempenho em condições de alta versus baixa sobre-

${ }^{5}$ No caso da aplicação original do PDP à memória (e.g., Jacoby, Toth, \& Yonelinas, 1993), as base-lines de completamento de fragmentos correspondentes a palavras novas (não apresentadas na fase de estudo) podem ser usadas como uma forma de verificar que o critério de resposta não mudou entre os testes de inclusão e exclusão. Especificamente, as base-lines deverão ser aproximadamente iguais nos testes de inclusão e exclusão. Diferenças nas base-lines sugerem violações na equivalência dos processos nos dois testes (inclusivo e exclusivo).

${ }^{6}$ Uma análise mais detalhada das questões ligadas à estimação de parâmetros e testes de fit não cabe no âmbito do presente texto pelo que se convida os leitores interessados a consultar Yonelinas (1997, 1999; Yonelinas, Regehr \& Jacoby, 1995; Buchner, Erdfelder \& Vaterrodt-Pltinnecke, 1995; Erdfelder, Faul \& Buchner, 1996) no caso do PDP; e Garcia-Perez (2011; ver também Roberts \& Pashler, 2000) para uma discussão formal e análise crítica destes tópicos. 
carga deveriam levar a uma dissociação de processos traduzida no decréscimo de $\mathrm{C}$ e na invariância de $\mathrm{A}$, uma vez que a escassez de recursos deve reduzir a possibilidade de processamento controlado mas não deve afectar o processamento automático. Por outro lado, uma das marcas características dos processos automáticos é a sua natureza associativa enquanto os processos controlados são melhor descritos por uma linguagem do pensamento governada por regras (Sloman, 1996). Neste sentido, previu-se que tarefas de natureza associativa como a respostas aprendidas por repetição ou primação de repetição deveriam levar à dissociação inversa. Ou seja, afectar as estimativas de A mas deixar as de C largamente invariantes através de diferentes condições de aprendizagem associativa. Ambas as dissociações de processos acima referidas têm sido recorrentemente encontradas na investigação usando o PDP (e.g., Jacoby, Toth \& Yonelinas, 1993; Hay \& Jacoby, 1996).

A ideia geral é que a obtenção deste tipo de dissociações envolvendo invariância de um dos processos seria extremamente improvável na presença de violações dos pressupostos acima referidos. Tal possibilidade torna-se ainda mais improvável quando estas dissociações são repetidamente encontradas num mesmo domínio de estudo. Pelo contrário, a recorrente falha em encontrar tais dissociações é indicador de violações severas dos pressupostos do PDP.

\section{Cuidados na aplicação do PDP}

Na aplicação do PDP são importantes alguns cuidados no sentido de não haver violações dos pressupostos que são potencialmente evitáveis. Designadamente, os materiais e instruções usados nas condições de inclusão e de exclusão devem ser tão semelhantes quanto possível de maneira a promover o mesmo grau de activação dos processos automáticos. No mesmo sentido, as instruções e materiais devem garantir a simetria de funcionamento dos processos controlados entre as condições de inclusão e exclusão. $\mathrm{Ou}$ seja, embora estes processos contribuam para a mesma resposta dos processos automáticos na condição de inclusão e se oponham a esta resposta na condição de exclusão, o grau desta contribuição deve ser igual em módulo apesar de se manifestar em sentidos opostos.

É igualmente importante usar tarefas que não sejam nem muito difíceis nem muito fáceis uma vez que os pressupostos do PDP são mais facilmente violados quando o desempenho dos participantes é muito elevado ou muito baixo. Por exemplo, se o desempenho na condição de exclusão for perfeito (zero erros) o cálculo de A leva a um valor nulo $(\mathrm{A}=0)$. Não porque deixe de haver contribuições automáticas mas devido a um artefacto matemático que decorre da forma como se calcula A e C (ver equações pag.14). 
$\mathrm{Na}$ prática isto quer dizer que os dados de participantes com desempenho perfeito não podem ser usados com o PDP. A remoção dos dados destes participantes da análise é uma prática tolerada sobretudo se for uma proporção pequena (relativamente ao total da amostra) e aproximadamente igual nos diversos níveis da variável independente. Mas a forma aconselhada de lidar com este problema é simplesmente ter um número de ensaios suficientemente grande para garantir (por pré-teste se necessário) que a probabilidade de um participante conseguir exercer um controlo perfeito em todos os ensaios seja minimizada (i.e., haver sempre erros) evitando-se (ou minimizando-se) assim a remoção de participantes da análise de dados.

Seguidamente veremos dois exemplos de aplicação do PDP que nos ajudarão a ilustrar como se traduzem na prática os cuidados acima referidos. Note-se que para usar o PDP com sucesso é necessário encontrar condições experimentais específicas que não violem os principais pressupostos do PDP e, desta forma, permitam a obtenção de estimativas de Controlo e Automaticidade independentes.

\section{Exemplos de Aplicação do PDP}

\section{PDP e estereótipos sociais}

No seguimento de trabalhos como o de Devine (1989) sobre as características automáticas e controladas dos estereótipos sociais, Payne (2001) propôs a tarefa hoje conhecida por shooter task em combinação com o PDP justamente para dissociar as componentes automática e controlada dos estereótipos sociais. Na aplicação original desta tarefa, Payne apresentou uma sequência de fotografias de armas (e.g., pistola) ou ferramentas (e.g., chave de fendas) cada uma imediatamente precedida pela fotografia de um caucasiano ou de um afro-americano. A tarefa dos participantes era indicar tão rápido quanto possível, para cada objecto apresentado, se se tratava de uma arma ou de uma ferramenta. Payne considerou que a condição de inclusão do seu estudo correspondia aos ensaios em que uma arma era antecedida pela fotografia de um afro-americano (quer o controlo decorrente da intenção de categorizar correctamente o objecto, quer o estereótipo do afro-americano que associa este grupo ao uso de armas levavam os participantes a responder "arma"). Enquanto os ensaios em que uma ferramenta era antecedida pela fotografia de um afro-americano correspondiam aos ensaios de exclusão (a resposta controlada opunha-se à associação estereotípica afro-americano arma). Com esta aplicação do PDP, Payne (estudo 1, 2001) mostrou que as fotografias de afro-americanos (por comparação com as de caucasianos) tiveram uma influência automática (derivada das associações estereotípicas) independente do processamento controlado. Este efeito diz-se automático 
porque a tendência para responder "arma" na presença das fotografias de afro-americanos (vs. fotografias de caucasianos) influenciou as respostas do sujeito quer esta convergisse ou se opusesse ao desempenho intencional dos participantes (i.e., categorizar correctamente os objectos em "armas" ou "ferramentas").

Este estudo de Payne (2001) é uma das primeiras aplicações do PDP fora do âmbito dos estudos puramente de memória e marca o início da investigação que tornou o PDP um verdadeiro instrumento geral de dissociação de processos

\section{PDP e julgamento na incerteza}

Muitas das decisões, escolhas e julgamentos do nosso dia-a-dia são feitas de forma intuitiva ou heurística. As inferências que realizamos em condições de incerteza surgem-nos naturalmente e sem esforço ou intenção e parecem decorrer de um conjunto de heurísticas ou atalhos cognitivos largamente automáticos que há muito são investigados em psicologia (e.g., Gilovich, Griffin \& Kahneman; 2002; Tversky \& Kahneman, 1982). Contudo, o julgamento humano não se resume a estas heurísticas. Nalgumas circunstâncias processos de decisão deliberados sobrepõem-se à intuição dominando as decisões. Num esforço de caracterização e compreensão da natureza destes dois processos e da forma com interagem entre si, Ferreira e colaboradores (2006) utilizaram o PDP. O objectivo era dissociar processos automáticos e controlados no domínio do julgamento na incerteza manipulando variáveis que distinguem conceptualmente os dois processos.

Para o efeito desenvolveu-se um conjunto de problemas directamente inspirados no tipo de problemas inferenciais historicamente usados na investigação em julgamento e decisão. As condições de inclusão e exclusão foram definidas não por instruções mas pela própria estrutura dos problemas (o que simplifica o procedimento uma vez que deixa de ser necessário instruir os participantes para se comportarem de forma diferente numa e noutra condição). Assim, cada participante respondia a um conjunto de problemas inferenciais, metade dos quais eram problemas de inclusão (i.e., problemas em que os julgamentos automáticos e controlados convergiam na mesma resposta) enquanto a outra metade eram problemas de exclusão (i.e., problemas em que os julgamentos controlados se opunham aos automáticos). As versões de inclusão e exclusão de cada problema eram exactamente iguais excepto no que dizia respeito aos dados considerados importantes para o processamento controlado que eram invertidos num dos casos (de forma a manter a simetria da estrutura do problema).

Assim, por exemplo um problema como o dos Engenheiros e Advogados (Kahneman \& Tversky, 1972) poderia ser usado como um problema 
de exclusão, uma vez que na sua forma original, processos controlados opõem-se aos automáticos:

Vários psicólogos entrevistaram um grupo de pessoas. O grupo incluía 30 engenheiros e 70 advogados. Os psicólogos prepararam um breve sumário da sua impressão relativa a cada entrevistado. A descrição seguinte foi escolhida aleatoriamente do conjunto de todas as descrições: O João tem 45 anos. É conservador, cuidadoso e ambicioso. Não mostra interesse por assuntos políticos e despende a maior parte do seu tempo livre nos seus muitos hobbies, que incluem carpintaria, velejar, e fazer puzzles matemáticos.

Qual das seguintes alternativas é mais provável?

a) O João é engenheiro.

b) O João é advogado.

Esta versão constitui a condição de exclusão uma vez que temos duas formas distintas de responder ao problema: um julgamento largamente automático com base na semelhança entre a descrição do João e o estereótipo de Engenheiro que fornece uma resposta imediata e espontânea para o problema: o João é Engenheiro (heurística da representatividade); e um julgamento controlado com base numa regra de amostragem que reconhece a importância de considerar o número relativo de engenheiros e advogados na estimativa da resposta mais provável (de facto, dado que a descrição foi escolhida aleatoriamente e que 7 em cada 10 são advogados a probabilidade de partida de ser um advogado é claramente superior à de ser engenheiro apenas 3 em cada 10 pessoas neste amostra são engenheiros). Os dois modos de processamento, automático e controlado estão assim em oposição.

Para criar a versão de inclusão deste problema basta inverter as probabilidade de partida (i.e., o texto passa a dizer que "...o grupo incluía 70 engenheiros e 30 advogados"). Consegue-se assim que a prevalência das respostas controlada e automática seja sensivelmente constante nas duas versões, com a diferença que os processos controlados de julgamento (neste caso a aplicação deliberada de uma regra de amostragem) convergem na mesma resposta dos automáticos (neste caso um julgamento baseado na heurística da representatividade) na versão de inclusão mas opõem-se a ela na versão de exclusão.

Após o desenvolvimento dos problemas nas suas versões inclusiva e exclusiva, todos os problemas foram pré-testados de forma a verificar que não eram nem demasiado difíceis nem demasiado fáceis. Só aqueles problemas que se revelaram moderadamente difíceis é que foram usados.

Por fim, realizaram-se vários estudos experimentais onde os participantes respondiam a séries destes problemas em diversas condições experimentais (Ferreira et al., 2006). Num dos estudos, foi pedido a metade dos 
participantes para responder aos problemas usando a sua intuição e sem perder muito tempo com nenhuma resposta em particular, enquanto a outra metade foi instruída para basear as suas respostas em reflexão racional. Os resultados mostraram que os participantes que baseavam as suas respostas em reflexão racional tinham um melhor desempenho nos problemas do que os participantes que usavam sobretudo a sua intuição, em especial nos problemas de exclusão. A aplicação do PDP permitiu verificar que esta superioridade se deveu a um maior processamento controlado da informação dos participantes da condição de reflexão racional em relação aos participantes da condição de resposta intuitiva. Por outro lado, a estimativa de processamento automático dos participantes das condições de resposta intuitiva e de reflexão racional manteve-se igual. As instruções afectaram unicamente os processos controlados de aplicação de regras deixando invariante a tendência para fazer julgamentos automáticos com base em heurísticas. O quer dizer, o que distingue os participantes instruídos para serem racionais dos instruídos a ser intuitivos não é a tendência para fazer julgamentos com base em heurísticas (nisto eles são virtualmente iguais), mas sim o controlo que os primeiros exercem sobre esta tendência, inibindo as respostas heurísticas e substituindo-as por respostas baseadas em regras (Ferreira et al., 2006; estudo 1). O padrão inverso de resultados, com uma manipulação experimental a afectar unicamente os processos automáticos deixando invariantes os controlados, foi igualmente obtido por Ferreira e colaboradores (2006; estudo 3).

\section{PDP e automaticidade}

As estimativas automática e controlada obtidas através do PDP podem ter diferentes significados conforme as tarefas e contextos em que o PDP é usado. Assim, ao contrário de abordagens que definem automatismo em termos de listas de características (e.g., intencionalidade, eficiência, controlabilidade, consciência; Bargh, 1994), o PDP implica que se comece por especificar qual o significado do controlo exercido numa dada tarefa (i.e., em que é que o recurso a um processamento controlado se traduzirá em termos de desempenho na tarefa). Recordemos, a este respeito, o exemplo dos doces anteriormente usado onde controlo foi definido como "não comer doces durante a semana", uma vez que era essa a intenção declarada da pessoa. Automatismo é depois definido como sendo a influência que guia as respostas dos participantes quando o controlo falha (no mesmo exemplo "comer doces durante a semana" decorrente da tentação que a pessoa tinha por doces). Outras características mais ou menos associadas aos processamentos automático e controlado (conforme o domínio de estudo e as abordagens teóricas adoptadas) são depois investigadas empiricamente, o que está 
em linha com a ideia de que a distinção entre automatismo e controlo não é uma questão de tudo ou nada mas antes uma questão de grau (Wegner \& Bargh, 1998).

Com efeito, uma das vantagens do PDP e da definição de automaticidade que incorpora é a sua capacidade para acomodar naturalmente resultados que podem surgir como paradoxais para as abordagens de automatismo baseadas na listagem de características de automatismo e de controlo (ver página 3).

Por exemplo, apesar das teorias clássicas considerarem processos automáticos como dificilmente controláveis, Stewart e Payne (2008) mostraram que a implementação de intenções para pensar de forma contra-estereotípica reduz o enviesamento automático mesmo em sobrecarga cognitiva. A utilização do PDP neste caso permitiu verificar que esta manipulação de intenção afectou A enquanto manteve $\mathrm{C}$ invariável, evidenciando um padrão de moderação distinto do encontrado (ou mesmo esperado) normalmente.

\section{Alternativas e limitações do PDP}

Conforme referido anteriormente, o PDP apresenta algumas limitações que resultam da necessidade de verificação dos seus pressupostos (i.e. sempre que estes são severamente violados o PDP não deve ser usado porque fornece estimativas erradas dos processos). Note-se, no entanto, que todos os procedimentos têm pressupostos cuja violação põe em causa a sua validade. Veja-se talvez o caso mais comum que é a tendência, em investigação experimental, para equacionar dissociações de tarefas como indicador de dissociação de processos. Tal estratégia, como vimos, baseia-se no pressuposto de que cada tarefa mede apenas um e um só processo. Ora se os pressupostos do PDP podem por vezes ser violados, o pressuposto da tarefa "pura" subjacente à dissociação de tarefas é quase sempre violado.

Uma outra limitação do PDP decorre do facto de se tratar de um modelo matemático extremamente simples, o que limita a possibilidade de realização de testes directos de fit das estimativas de $\mathrm{C}$ e A. Contudo esta simplicidade é provavelmente a razão pela qual o PDP tem tido uma utilização tão vasta e diversificada na investigação de processos automáticos e controlados em psicologia. Estão, no entanto, disponíveis outros modelos matemáticos mais complexos (e.g., o modelo QUAD proposto por Conrey, Sherman, Gawronski, Hungenberg, \& Groom, 2005; o modelo ABC de Stahl \& Degner, 2007; o modelo conjoint recognition de Brainerd, Reyna, \& Monjardin, 1999) que não só envolvem mais parâmetros e diferentes interpretações destes parâmetros, como implicam a obtenção de mais dados empíricos. 
Testes de fit dos seus parâmetros tornam a validação destes modelos menos dependente da obtenção de padrões de dissociação específicos como acontece no caso do PDP. O uso destes modelos em detrimento do PDP decorrerá, para cada investigador, de considerações teóricas (e.g., número de processos independentes que um modelo parcimonioso do comportamento humano deve incluir) assim como de análises custo-benefício da sua utilização.

\section{Conclusões}

As teorias dualistas de processamento baseiam-se na ideia de que o comportamento humano resulta simultaneamente de processos controlados e automáticos. No entanto, é difícil testar estes modelos sem meios para quantificar a influência específica destes processos. Instrumentos metodológicos como o PDP fornecem uma solução para este problema permitindo aos investigadores separar e comparar processos automáticos e controlados. Uma outra contribuição do PDP (e modelos semelhantes) é o facto de a sua utilização forçar os investigadores a explicitarem as suas definições de controlo e automatismo e a serem mais específicos na formulação das suas hipóteses. Mais do que afirmações gerais sobre qual dos processos, automático ou controlado, domina o comportamento humano, com o PDP é possível testar empiricamente a influência de ambos os processos em diferentes domínios de actividade e para diferentes tipos de comportamento humano.

\section{Referências}

Anderson, J. R., \& Bower, G. H. (1973). Human associative memory. Washington: D.C.: Winston.

Bargh, J. A. (1994). The Four Horsemen of automaticity: Awareness, efficiency, intention, and control in social cognition. In R. S. Wyer, Jr., \& T. K. Srull (Eds.), Handbook of social cognition (2nd ed., pp. 1-40). Hillsdale, NJ: Erlbaum.

Bishara, A. J., (2005). Control and accessibility bias in single and multiple anchoring effects. Unpublished doctoral dissertation, Washington University.

Brainerd, C. J., Reyna, V. F., \& Mojardin, A. H. (1999). Conjoint recognition. Psychological Review, 106(1), 160-179.

Buchner, A., Erdfelder, E., \& Vaterrodt-Pltinnecke, B. (1995). Toward unbiased measurement of conscious and unconscious memory processes within the process dissociation framework. Journal of Experimental Psychology: General, 124, 137-160.

Conrey, F. R., Sherman, J. W., Gawronski, B., Hugenberg, K., \& Groom, C. (2005). Separating multiple processes in implicit social cognition: The Quad-Model of 
implicit task performance. Journal of Personality and Social Psychology, 89, 469-487.

Conway, P., \& Gawronski, B. (2013). Deontological and utilitarian inclinations in moral decision-making: A process dissociation approach. Journal of Personality and Social Psychology, 104, 216-235.

Devine, P. G. (1989). Stereotypes and prejudice: Their automatic and controlled components. Journal of Personality and Social Psychology, 56, 5-18.

Erdfelder, E., Faul, E, \& Buchner, A. (1996). G-Power: A general power analysis program. Behavior Research Methods, Instruments and Computers, 28, 1-11.

Fazio, R. H., Jackson, J. R., Dunton, B. C., \& Williams, C. J. (1995). Variability in automatic activation as an unobstrusive measure of racial attitudes: A bona fide pipeline? Journal of Personality and Social Psychology, 69(6), 1013-1027.

Ferreira, M. B., Garcia-Marques, L., Sherman, S. J., \& Sherman, J. W. (2006). Automatic and controlled components of judgment and decision making. Journal of Personality and Social Psychology, 91(5), 797-813.

Ferreira, M. B., Garcia-Marques, L., Hamilton, D. L., Ramos, T., Uleman, J., \& Jerónimo, R. (2012). On the relation between spontaneous trait inferences and intentional inferences: An inference monitoring hypothesis. Journal of Experimental Social Psychology, 48, 1-12.

García-Pérez, M. A. (1994). Parameter estimation and goodness-of-fit testing in multinomial models. British Journal of Mathematical and Statistical Psychology, 47, 247-282. doi: 10.1111/j.2044-8317.

Garcia-Marques, T., \& Ferreira, M. B. (no prelo). Paradigma de suporte ao Procedimento de Dissociação de Processsos. Laboratório de Psicologia.

Gilovich, T., Griffin, D., \& Kahneman, D. (Eds.). (2002). Heuristics and biases: The psychology of intuitive judgment. Cambridge, England: Cambridge University Press.

Graf, P., \& Mandler, G. (1984). Activation makes words more accessible, but not necessarily more retrievable. Journal of Verbal Learning and Verbal Behavior, 23(5), 553-568.

Greenwald, A. G., McGhee, D. E., \& Schwartz, J. L. K. (1998). Measuring individual differences in implicit cognition: the implicit association test. Journal of Personality and Social Psychology, 74(6), 1464-1480.

Haddock, G., Zanna, M. P., \& Esses, V. M. (1993). Assessing the structure of prejudicial attitudes: The case of attitudes toward homosexuals. Journal of Personality and Social Psychology, 65(6), 1105-1118.

Hay, J. F., \& Jacoby, L. L. (1996). Separating habit and recollection: Memory slips, process dissociations and probability matching. Journal of Experimental Psychology: Learning, Memory and Cognition, 22, 1323-1335

Hintzman, D. L. (1990). Human learning and memory: Connections and dissociations. Annual Review of Psychology, 41(1), 109-139.

Jacoby, L. L. (1991). A process dissociation framework: Separating automatic from intentional uses of memory. Journal of Memory and Language, 30(5), 513-541. 
Jacoby, L. L. (1998). Invariance in automatic influences of memory: Toward a user's guide for the process-dissociation procedure. Journal of Experimental Psychology: Learning, Memory, and Cognition, 24(1), 3-26.

Jacoby, L. L., Kelley, C. M., Brown, J., \& Jasechko, J. (1989). Becoming famous overnight: Limits on the ability to avoid unconscious influences of the past. Journal of Personality and Social Psychology, 56, 326-338.

Jacoby, L. L., Toth, J. P., \& Yonelinas, A. P. (1993). Separating conscious and unconscious influences of memory: Measuring recollection. Journal of Experimental Psychology: General, 122(2), 139-154.

Kahneman, D., \& Tversky, A., (1972). Subjective probability: A judgment of representativeness. Cognitive Psychology, 3, 430-454.

Logan, G. D. (1980). Attention and automaticity in Stroop and priming tasks: Theory and data. Cognitive Psychology, 12, 523-553.

Logan. G. D., \& Cowan, W. B. (1984). On the ability to inhibit thought and action: A theory of an act of control. Psychological Review, 91, 295-327.

Mandler, G. (1980). Recognizing: The judgment of previous occurrence. Psychological Review, 87, 252-271.

McConahay, J. B. (1986). Modern racism, ambivalence, and the Modern Racism Scale. In J. F. Dovidio \& S. L. Gaertner (Eds.), Prejudice, discrimination, and racism (pp. 99-125). Orlando: Academic Press.

Neely, J. H. (1977). Semantic priming and retrieval from lexical memory: Roles of inhibitionless spreading activation and limited-capacity attention. Journal of Experimental Psychology: General, 106, 226-254.

Payne, B. K. (2001). Prejudice and perception: the role of automatic and controlled processes in misperceiving a weapon. Journal of Personality and Social Psychology, 81(2), 181-192.

Payne, B. K. (2005). Conceptualizing Control in Social Cognition: How executive control modulates the expression of automatic stereotyping. Journal of Personality and Social Psychology, 89, 488-503.

Payne, B. K. (2008). What mistakes disclose: A process dissociation approach to automatic and controlled processes in social psychology. Social and Personality Psychology Compass, 2(2), 1073-1092.

Payne, B. K., \& Bishara, A. J. (2009). An integrative review of process dissociation and related models in social cognition. European Review of Social Psychology, 20(1), 272-314.

Posner, M. I., \& Snyder, C. R. (1975). Attention and cognitive control. In R. L. Solso (Ed.), Information processing and cognition (pp. 55-85). Hillsdale, NJ: Erlbaum.

Reingold, E. M., \& Merikle, P. M. (1990). On the inter-relatedness of theory and measurement in the study of unconscious processes. Mind \& Language, 5(1), 9-28.

Roberts, S., \& Pashler, H. (2000). How persuasive is a good fit? A comment on theory testing. Psychological Review, 107, 358-367.

Schacter, D.L., Chiu, C.Y.P., \& Ochsner, K.N. (1993). Implicit memory: A selective review. Annual Review of Neuroscience, 16, 159-182. 
Schneider, W., \& Shiffrin, R. M. (1977). Controlled and automatic human information processing: Detection, search, and attention. Psychological Review, 84, 1-66.

Shiffrin, R. M., \& Dumais, S. T. (1981). The development of automatism. In J. R. Anderson (Ed.), Cognitive skills and their acquisition (pp. 111-140). Hillsdale, N.J.: Erlbaum.

Shiffrin, R. M., \& Schneider, W. (1977). Controlled and automatic human information processing: II. Perceptual learning, automatic attending, and a general theory. Psychological Review, 84, 127-190.

Shiv, B., \& Fedorikhin, A. (1999). Heart and mind in conflict: The interplay of affect and cognition in consumer decision making. Journal of Consumer Research, 26, 278-292.

Sloman, S. A. (1996). The empirical case for two systems of reasoning. Psychological Bulletin, 119(1), 3-22.

Stahl, C., \& Degner, J. (2007). Assessing automatic activation of valence: A multinomial model of EAST performance. Experimental Psychology, 54, 99-112.

Stewart, B. D., \& Payne, B. K. (2008). Bringing automatic stereotyping under control: Implementation intentions as efficient means of thought control. Personality and Social Psychology Bulletin, 34, 1332-1345.

Tversky, A., \& Kahneman, D. (1982). Evidential impact of base rates. In D. Kahneman, P. Slovic, \& A. Tversky (Eds.), Judgment under uncertainty: Heuristics and biases (pp. 117-128). New York, NY: Cambridge University Press.

Tulving, E., Schacter, D. L., \& Stark, H. A. (1982). Priming effects in word-fragment completion are independent of recognition memory. Journal of Experimental Psychology: Learning, Memory, and Cognition, 8(4), 336-342.

Wegner, D. M., \& Bargh, J. A. (1998). Control and automaticity in social life. In D. T. Gilbert, S. T. Fiske, \& G. Lindzey (Eds.), Handbook of social psychology (pp. 446-496). (4th ed). New York: McGraw-Hill Vol. 1.

Yonelinas, A. P. (1997). Recognition memory ROCs for item and associative information: The contribution of recollection and familiarity. Memory and Cognition, 25 (6), 747-763.

Yonelinas, A. P. (1999). The contribution of recollection and familiarity to recognition and source memory: An analysis of receiver operating characteristics and a formal model. Journal of Experimental Psychology: Learning, Memory, and Cognition, 25 (6), 1415-1434.

Yonelinas, A. P., \& Jacoby, L. L. (2012). The process-dissociation approach two decades later: Convergence, boundary conditions, and new directions. Memory \& Cognition, 40, 663-680.

Yonelinas, A. P., Regehr, G., \& Jacoby, L. L. (1995). Incorporating response bias in a dual-process theory of memory. Journal of Memory and Language, 34, 821-835 . 